\title{
Estudo da Inibição de Corrosão do aço carbono em ácido clorídricona presença de mangiferina
}

\section{Study of the Corrosion Inhibition of Carbon Steel in acidic chloride by Mangiferin}

\author{
Jorge Edson Pinheiro dos Santos ${ }^{1}$, Ana Paula Rodrigues do Nascimento Barroso ${ }^{2}$, \\ Marcelo Monteiro Valente Parente ${ }^{3}$, Caroline de Goes Sampaio ${ }^{4}$
}

\author{
${ }^{1,2}$ Laboratório de Inspeção e Analise de Falhas - LIAF-IFCE: 61.939-140, Maracanaú, CE \\ e-mail: jepsa-015@hotmail.com; appaulabarroso@gmail.com \\ ${ }^{3}$ IFCE: 61600-000, Caucaia, CE \\ e-mail: marceloparente2@gmail.com \\ ${ }^{4}$ IFCE: 61.939-140, Maracanaú, CE \\ e-mail: carol-quimica@hotmail.com
}

\section{RESUMO}

Controlar, ou inibir cineticamente o processo corrosivo tem sido um grande desafio para a ciência. Desta forma, tem-se buscado estudar os diversos tipos de interações entre material e meio. A utilização de inibidores constitui um dos métodos usuais de proteção contra a corrosão. No entanto, há uma preocupação crescente sobre a toxicidade da maior parte dos inibidores de corrosão. Vários trabalhos científicos têm sido realizados que comprovam a ação inibidora de extratos de plantas frente à corrosão de metais. Este estudo tem por objetivo verificar efeito inibidor da Mangiferina, substância obtida a partir da extração do caule da planta Mangifera indica L, frente ao processo corrosivo em aço carbono. Para isso, foram realizados ensaios de perda de massa coadjuvados com técnicas eletroquímicas em meio de ácido clorídrico. Os resultados obtidos mostraram que aço carbono exposto neste meio adicionado de Mangiferina apresentou uma redução no processo de perda de massa e um comportamento eletroquímico superior em relação às amostras de aço carbono expostas no mesmo meio na ausência dessa substância em estudo. Estes resultados sugerem que a Mangiferina apresenta ação inibidora no processo corrosivo do aço carbono nas condições estudadas.

Palavras-chaves: Corrosão, Inibidor, Mangiferina.

\begin{abstract}
To control or to kinetically inhibit corrosive process has become a major challenge for science. Because of this, researchers have tried to study the various types of interactions between the material and the environment. The use of inhibitors is one of the usual methods of corrosion protection. However, there is growing concern about the toxicity of most corrosion inhibitors. Several studies have been conducted that demonstrate the inhibitory action of plants' extracts on the corrosion of metals. This study aims to verify the inhibitory effect of Mangiferin, substance obtained from the extraction of the plant stem of Mangifera indica L, against the corrosive process in carbon steel. In this study, weight loss tests were carried out assisted by electrochemical techniques in acidic chloride. The results showed that carbon steel exposed in this medium containing Mangiferin had a reduction in mass loss process and a higher electrochemical behavior relative to the carbon steel samples exposed in the same medium in the absence of Mangiferin. These results suggest that Mangiferin shows inhibitory action on the corrosion process of carbon steel in the studied conditions.
\end{abstract}

Keywords: Corrosion, Inhibitor, Mangiferin.

\section{INTRODUÇÃO}

A maior parte do processo de corrosão dos metais é resultante de reações eletroquímicas entre o meio e a sua superfície. Desta forma, as metodologias de proteção se baseiam em inibir cineticamente essas reações que 
vem se tornado um grande desafio para a ciência ao longo dos anos [1][2].

No mundo atual, mesmo em meio a grande busca por novos materiais com propriedades distintas, o aço carbono continua sendo o mais importante, devido as suas boas propriedades mecânicas e seu baixo custo [3]. No entanto, a sua utilização se torna limitada devido sua alta suscetibilidade ao processo corrosivo. Desta forma, se faz necessário o uso de métodos que venham controlar ou inibir este efeito.

Atualmente, as inovações nesta área estão associadas ao desenvolvimento de novos materiais, tais como o desenvolvimento de novas ligas que sejam mais resistência a corrosão e de baixo custo, desenvolvimentos de tintas impermeáveis e inteligentes e desenvolvimento de inibidores de corrosão[4].

Pode ser visto que, dependendo da aplicação, a utilização de inibidores é um dos melhores métodos de proteção contra a corrosão em metais[5][6]. No entanto, há uma preocupação crescente sobre a toxicidade desse inibidores. $\mathrm{O}$ efeito tóxico, não afeta somente os organismos vivos, mas também contamina o ambiente que, consequentemente, pode gerar problemas irreversíveis a o ecossistema afetado[6][7].

Com isso, nota-se a necessidade do uso de inibidores de corrosão naturais, que não agridam o meio ambiente. Inibidores desta classe são obtidos, principalmente, a partir de produtos naturais, tais como extratos de plantas [8]. Recentemente, vários estudos têm sido realizados sobre a inibição da corrosão de metais a partir de produtos obtidos de extrato de planta [9][10]. Como exemplo, pode ser citado o trabalho realizado pelos autores QURAISHI, M.A., et al que traz resultados positivos da ação inibidora do extrato da casca de Murraya koenigii [12].

A Mangiferina (2-C-b-D-glicopiranosil-1,3,6,7-tetrahidroxi-xantona) é um composto polifenólico amplamente encontrado em pteridófitas e angiospemas, principalmente nas famílias Gentianaceae e Iridaceae, presentes predominantemente nas folhas e na casca do caule. Em mangueira, mangiferina é um dos constituintes fenólicos majoritarios, podendo ser detectada nas folhas, casca do caule, fruto e raízes[14].

A Mangiferina apresenta um grande potencial antioxidante, pois apresenta uma capacidade elevada de absorção de radicais oxigênio, devido ao fato de apresentar zonas de radicais hidroxila. Relatos apresentados na literatura[13] mostram que a capacidade antioxidante da mangiferina é mais potente que a do ácido ascórbico e da vitamina E. A figura 01 está apresentada a sua estrutura química.

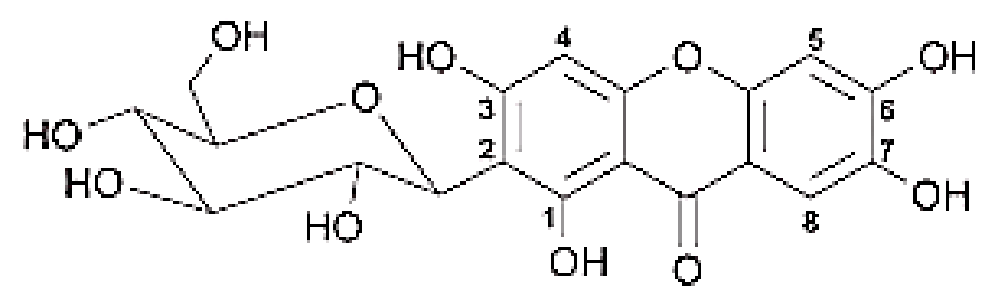

Figura 01: Estrutura química da Mangiferina [14]

Portanto, este trabalho tem como objetivo prioritário verificar a ação inibidora da Mangiferina em meio ácido.

\section{MATERIAIS E MÉTODOS}

A substância Mangiferina utilizada nesse trabalho foi cedida pelo Laboratório de Química Orgânica da Universidade Federal do Ceará - UFC. A mesma foi obtida a partir de extração do caule da planta Mangifera indica L. (mangueira), utilizando o etanol como solvente extrator. O procedimento de obtenção e purificação da Mangiferina está devidamente apresentada na literatura[11].

Para avaliar o efeito inibidor de corrosão da Mangiferina, foi utilizada solução aquosa de $\mathrm{HCl} 0,5$ mol. $L^{-1}$ na ausência e presença de Mangiferina, nas concentrações 100 e 300 ppm. Na condição ausente de Mangiferina, convencionou-se o parâmetro de concentração de 0 ppm. A adição da Mangiferina no preparo das soluções de testes foi realizada através de diluições a partir de seu extrato alcoólico de concentração de 10.000 ppm com 99,8\% de pureza. Como substrato, utilizou-se a liga de aço-carbono 1010 que foi confeccionada e tratada de acordo com os ensaios que a mesma foi submetida. Neste trabalho, a denominação para esta liga será apenas de aço-carbono.

Nos ensaios de perda de massa, foram utilizadas amostras de aço-carbono de dimensões ( $25 \mathrm{~mm}$ x 20 $\mathrm{mm} \times 1 \mathrm{~mm}$ ). As mesmas passaram por processo de lixamento com lixas d'agua de granulometria de 100 mesh utilizando politriz com rotação aproximada de $300 \mathrm{rpm}$. Posteriormente, as superfícies foram lavadas 
com água deionizada e etanol e secas com auxílio de ar quente. O procedimento adotado para este ensaio foi o mesmo apresentado na literatura[16], onde é descrito as dimensões e tratamento das superfícies em estudo. As amostras foram imersas em solução de $\mathrm{HCl}\left(0,5 \mathrm{~mol} \cdot \mathrm{L}^{-1}\right)$ até um intervalo de tempo de 144 horas na temperatura ambiente na ausência e presença Mangiferina nas concentrações de 100 e 300 ppm. A perda de massa foi determinada, gravimetricamente, empregando uma balança analítica com precisão de $0,1 \mathrm{mg}$. Os ensaios foram realizados em triplicatas. A eficiência de inibição (n\%) foi determinada pela Equação 1:

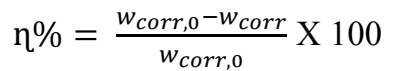

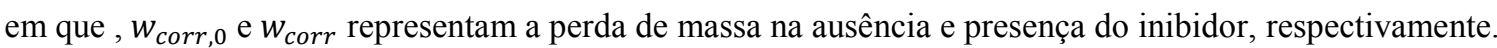

Para os ensaios eletroquímicos, as amostras de aço-carbono foram devidamente cortadas e conectadas a um fio de cobre encapado por intermédio de solda e, posteriormente, embutidas a frio, utilizando resina do tipo epóxi. As superfícies utilizada para o estudo foram lixadas com lixas d'água de granulometria de 100 até 600 mesh utilizando politriz com rotação aproximada de $300 \mathrm{rpm}$ até o desaparecimento dos traços da lixa anterior. Posteriormente, as superfícies foram lavadas com água deionizadas e etanol. Em seguida foram secos com fluxo de ar quente, conforme apresentado na literatura ${ }^{[16]}$. As amostras (Eletrodo de Trabalho) tiveram as suas bordas isoladas com esmalte, para evitar infiltração. Após a secagem, as áreas expostas foram devidamente medidas e calculadas.

Os ensaios eletroquímicos foram realizados utilizando um potenciostato/galvanostato AUTOLAB modelo PGSTAT 128N. O shoftware utilizado para o monitoramento foi o NOVA 1.8. Foi utilizada uma célula padrão de três eletrodos: o eletrodo $\mathrm{Ag} / \mathrm{AgCl} / \mathrm{KCl}$ saturado como eletrodo de referência, um contraeletrodo de platina, com área exposta superior a três vezes a do eletrodo de trabalho e o eletrodo de trabalho, propriamente dito. Os ensaios foram realizados em célula eletroquímica de capacidade de $250 \mathrm{~mL}$, contento a solução $\mathrm{HCl}$ 0,5 mol.L $\mathrm{L}^{-1}$ na ausência e presença de Mangiferina, nas concentrações de 100 e 300 ppm. Os ensaios são todos independentes. Após cada ensaio a superfície do eletrodo de trabalho era novamente preparado para ser utilizado em outro novo ensaio.

Inicialmente, foi realizado o ensaio de estabilização dos Potenciais de Circuito Aberto (PCAs) para verificar o tempo em que se estabilizava o sistema. Neste ensaio foi monitorado o tempo com relação à variação do PCAs.

Posteriormente, os ensaios realizados foram os de Espectroscopia de Impedância Eletroquímica (EIE). Nesse foi aplicado uma perturbação de amplitude fixa de $10 \mathrm{mV}$ ao PCA na ausência e presença de Mangiferina nas concentrações de 100 e 300 ppm. A faixa de frequência variante foi de $40 \mathrm{kHz}$ a $2,5 \mathrm{mHz}$. Os ensaisos de EIE foram interrompidos após a instabilização do sistema em frequências mais baixas. Neste trabalho, os diagramas de impedância estão apresentados no modelo de Bode e Nyquist.

Os últimos ensaios realizados, foram os de Polarização Potenciodinâmica onde a varredura iniciou-se a partir de uma sobrevoltagem de $-200 \mathrm{mV}$ a partir do PCA. Antes de iniciar o ensaio, o sistema foi estabilizado por 10 minutos. A taxa de varredura foi de $2,0 \mathrm{mV} \cdot \mathrm{s}^{-1}$.

Todos os resultados eletroquímicos tiveram seus resultados ajustados de acordo com a área de cada eletrodo de trabalho. Para fins de validação, todos os ensaios eletroquímicos foram realizados em duplicata.

\section{RESULTADOS}

Os ensaios foram todos realizados seguindo a metodologia já estabelecida nas concentrações de 0,100 e 300 ppm. Para o teste de perda de massa foi gerado os resultados expostos na figura 02. 


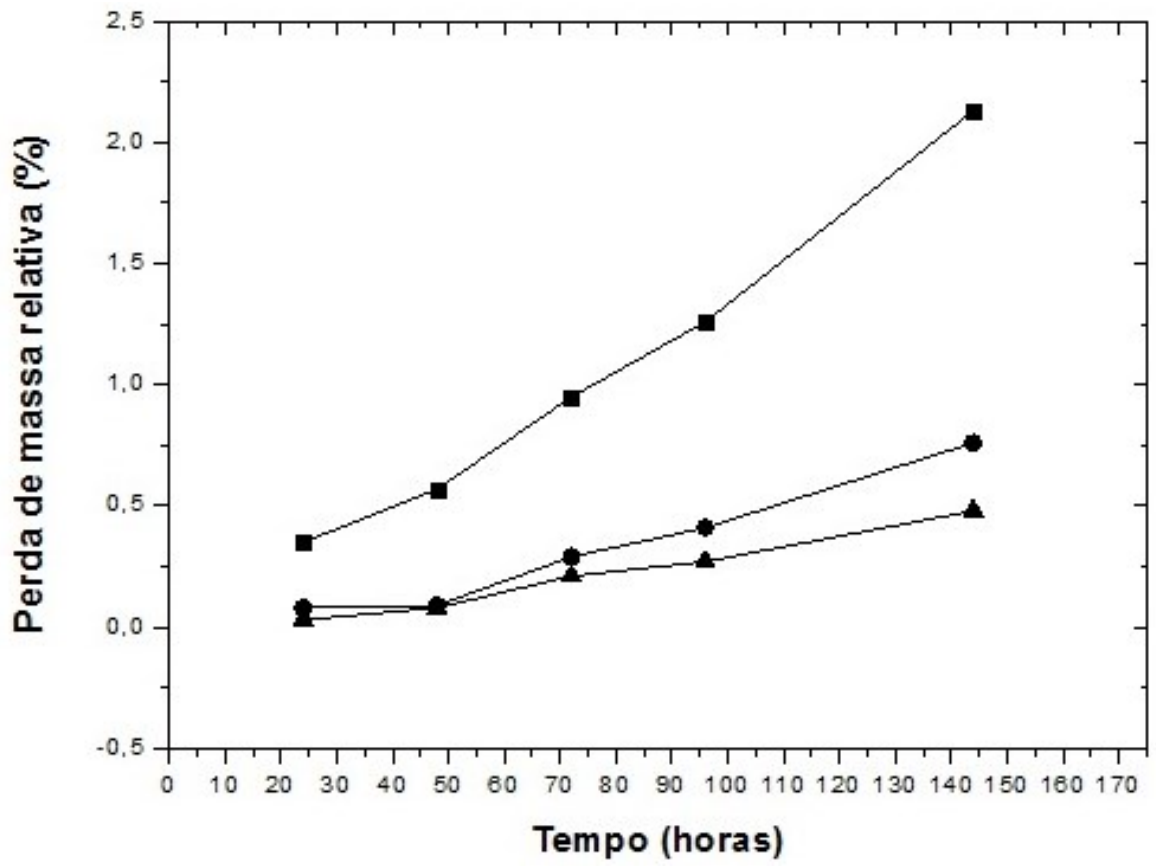

Figura 02: Ensaio de perda de massa para aço carbono em ácido clorídrico $0,5 \mathrm{~mol} . \mathrm{L}^{-1}$ na ausência e presença de extrato etanólico de Mangiferina nas concentrações de 100 e 300 ppm à temperatura ambiente.

Nos ensaios de monitoramento dos PCAs com o tempo, as amostras foram imersas nas soluções ácidas na ausência e adicionadas de Mangiferina em concentrações especificas (0, 100ppm e 300ppm), respectivamente. Na figura 03, está exposto o monitoramento dos potenciais de circuito aberto (PCA) com um tempo de imersão até 60 minutos.

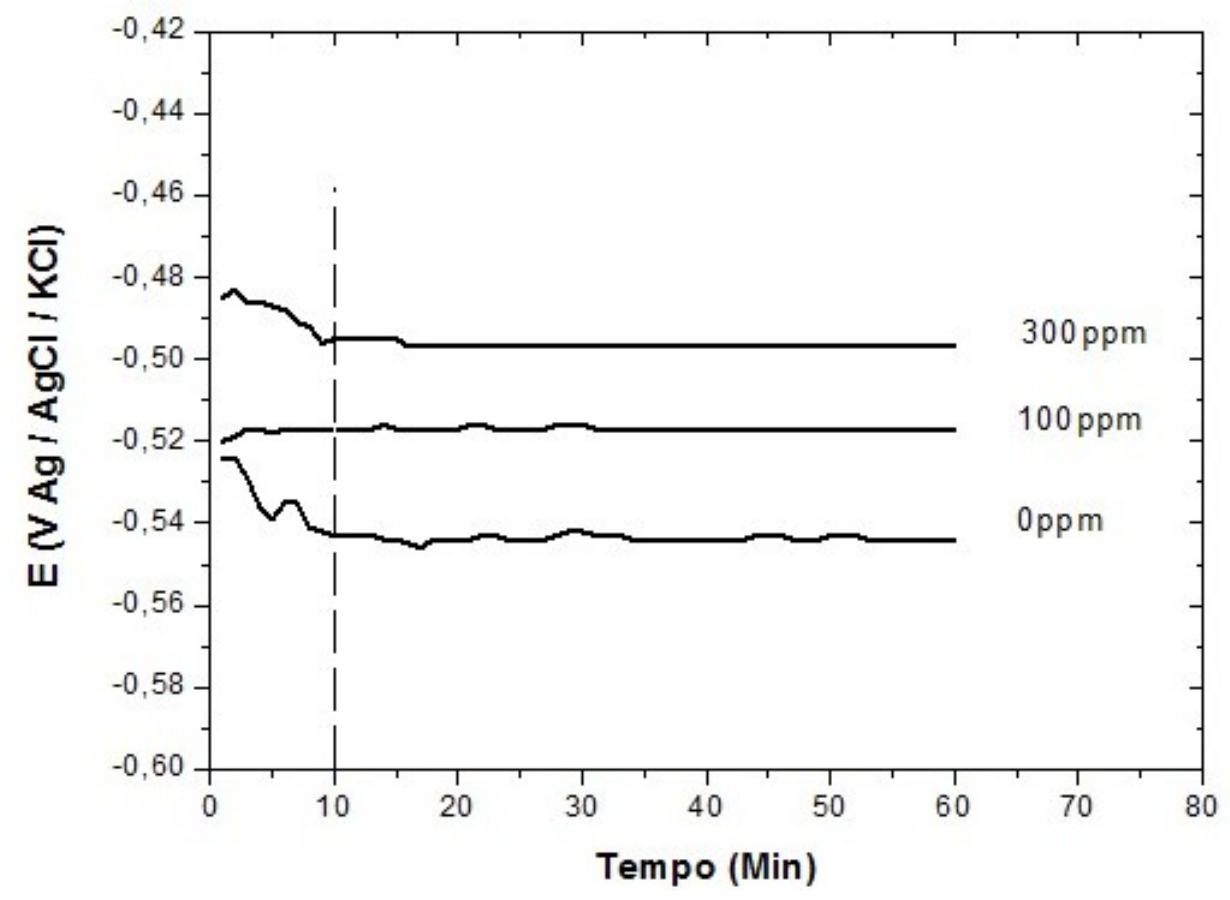

Figura 03: monitoramento do potencial de circuito aberto para aço carbono em ácido clorídrico 0,5 mol. $\mathrm{L}^{-1}$ na ausência e presença de extrato etanólico de Mangiferina nas concentrações de 100 e 300 ppm à temperatura ambiente.

Os ensaios EIE foram realizados após 10 minutos de estabilização. Nas figuras 04 e 05 estão apresentados os resultados nos diagramas de Bode e Nyquist respectivamente, obtidos para este ensaio nas concentrações de 100 e 300 ppm, sempre levando como referência a concentração sem acréscimo de Mangiferina. 

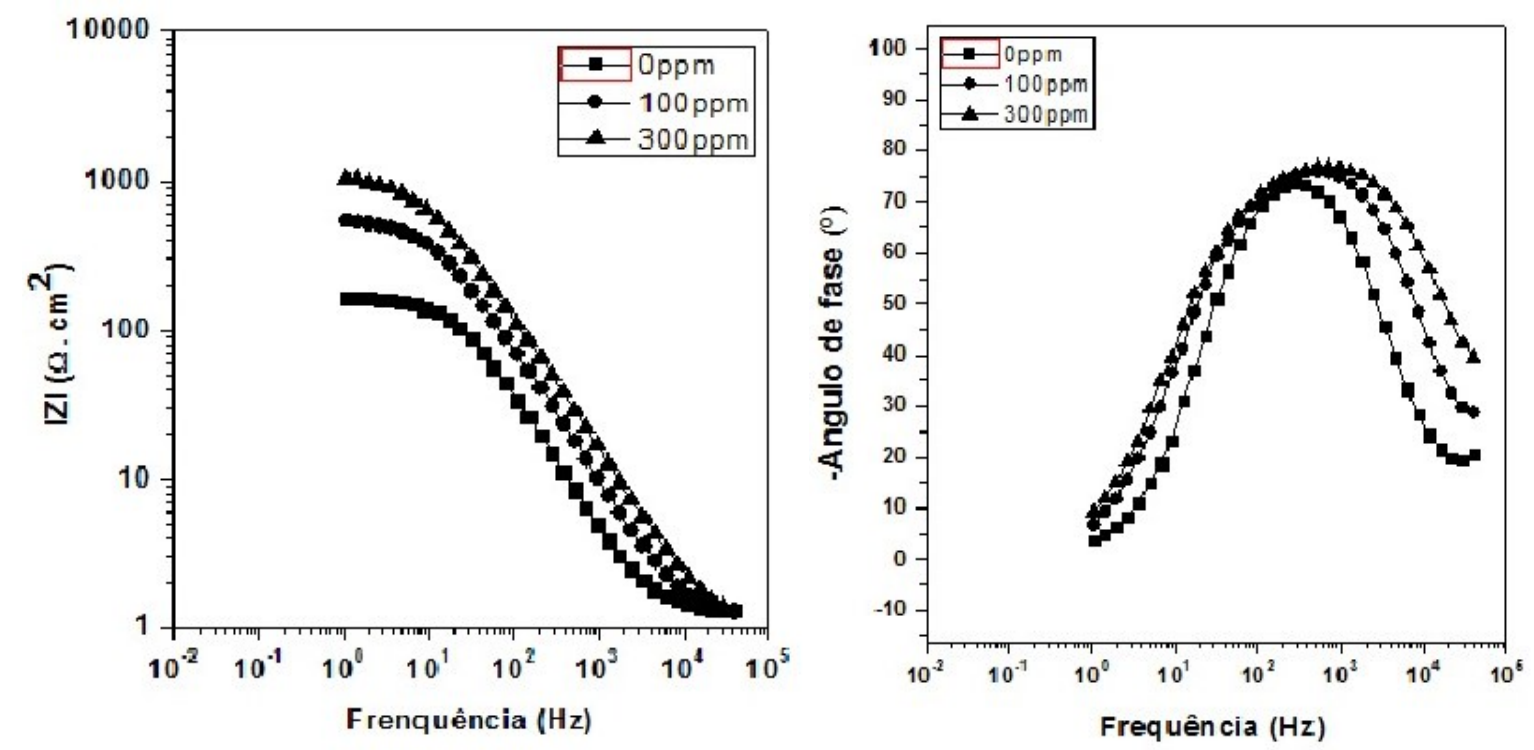

a)

b)

Figura 04: Diagrama de Bode obtido para aço-carbono em ácido clorídrico $0,5 \mathrm{~mol} \cdot \mathrm{L}^{-1}$ na ausência e presença de Mangiferina nas concentrações de 100 e 300 ppm à temperatura ambiente. a) módulo da impedância versus Frequência b) ângulo de fase versus Frequência.

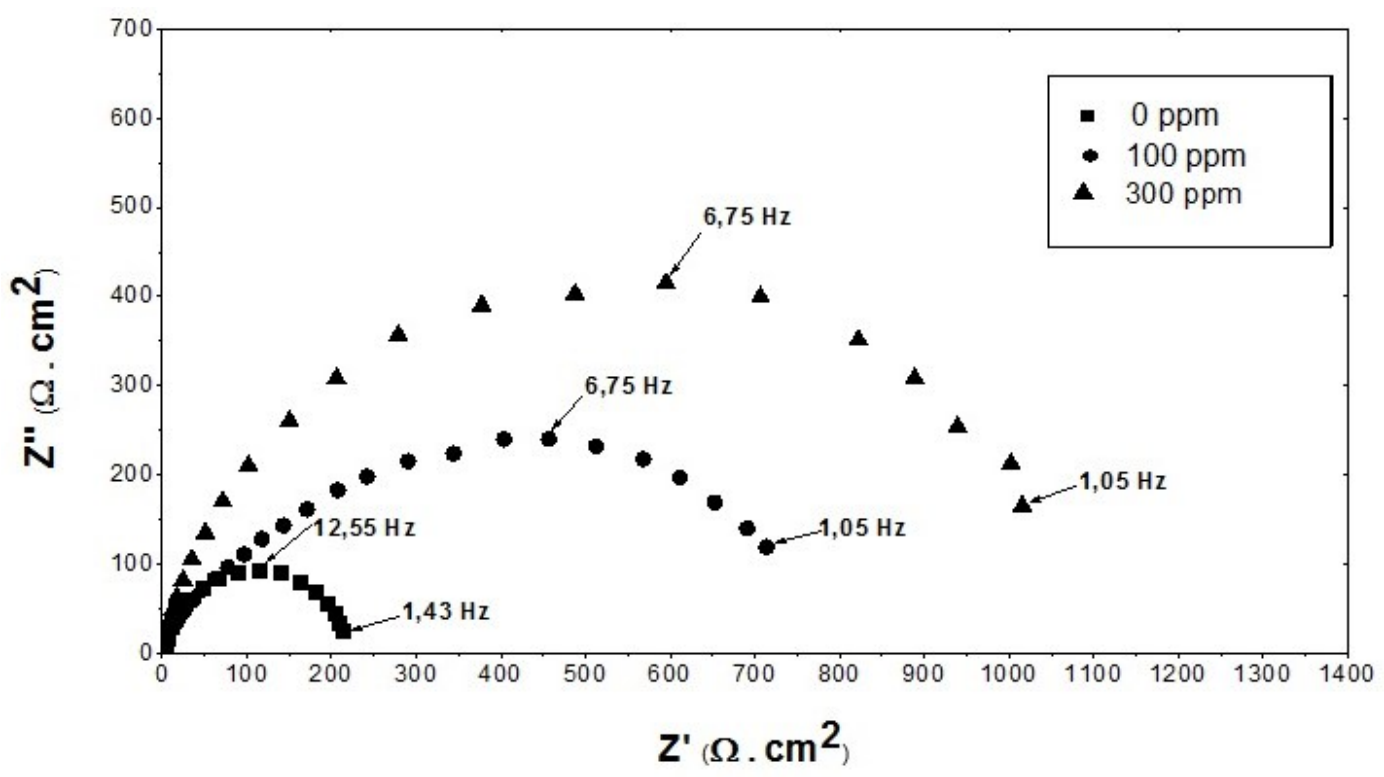

Figura 05: Diagrama de Nyquist obtido para aço-carbono em ácido clorídrico $0,5 \mathrm{~mol} \cdot \mathrm{L}^{-1}$ na ausência e presença de Mangiferina nas concentrações de 100 e 300 ppm à temperatura ambiente.

Valores de Resistência a Transferência de Carga (Rct) foram obtidos através da extrapolação do arco capacitivo até o eixo real. Na figura 06 (a) estão relacionados os valores de Rct com a concentração de Mangiferina. Também foram calculados valores de Capacitancia (C) da interface eletrodo solução de acordo com a frequência no ponto máximo do arco capacitivo. Na figura 06 (b) estão relacionados os valores de $\mathrm{C}$ com a concentração de Mangiferina. Os resultados estão apresentados na figura abaixo. 


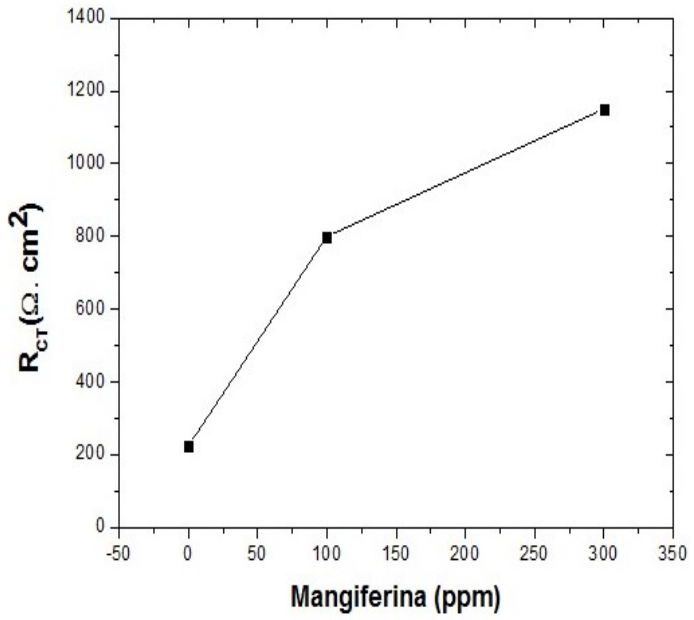

a)

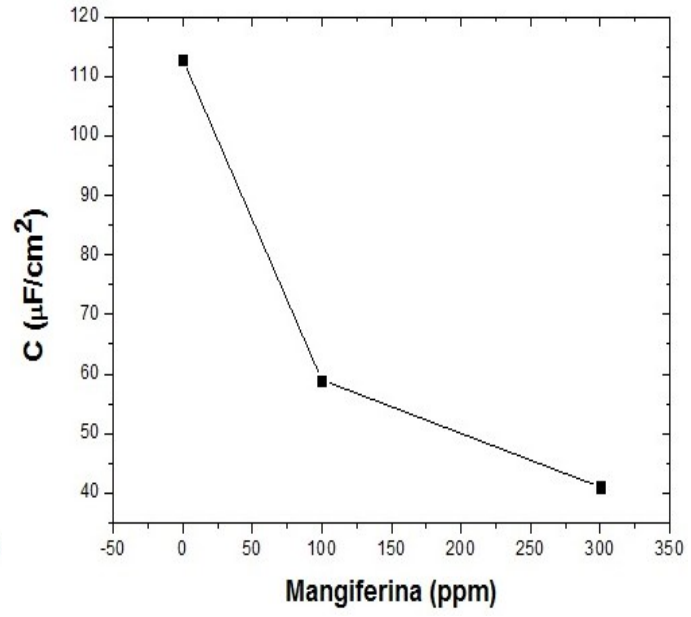

b)

Figura 06: Valores de Resistência a transferência de carga (Rct) e Capacitância (C) obtidos para aço-carbono em ácido clorídrico $0,5 \mathrm{~mol} \cdot \mathrm{L}^{-1}$ na ausência e presença de Mangiferina nas concentrações de 100 e $300 \mathrm{ppm}$ à temperatura ambiente. a) Rct versus concentração de Mangiferina b) Capacitância versus concentração de Mangiferina.

Os ensaios de polarização potenciodinâmica foram realizados após 10 minutos de estabilização. $\mathrm{Na}$ figura 07, estão apresentados os resultados obtidos para estes ensaios nas concentrações de 0, 100 e 300 ppm de Mangiferina.

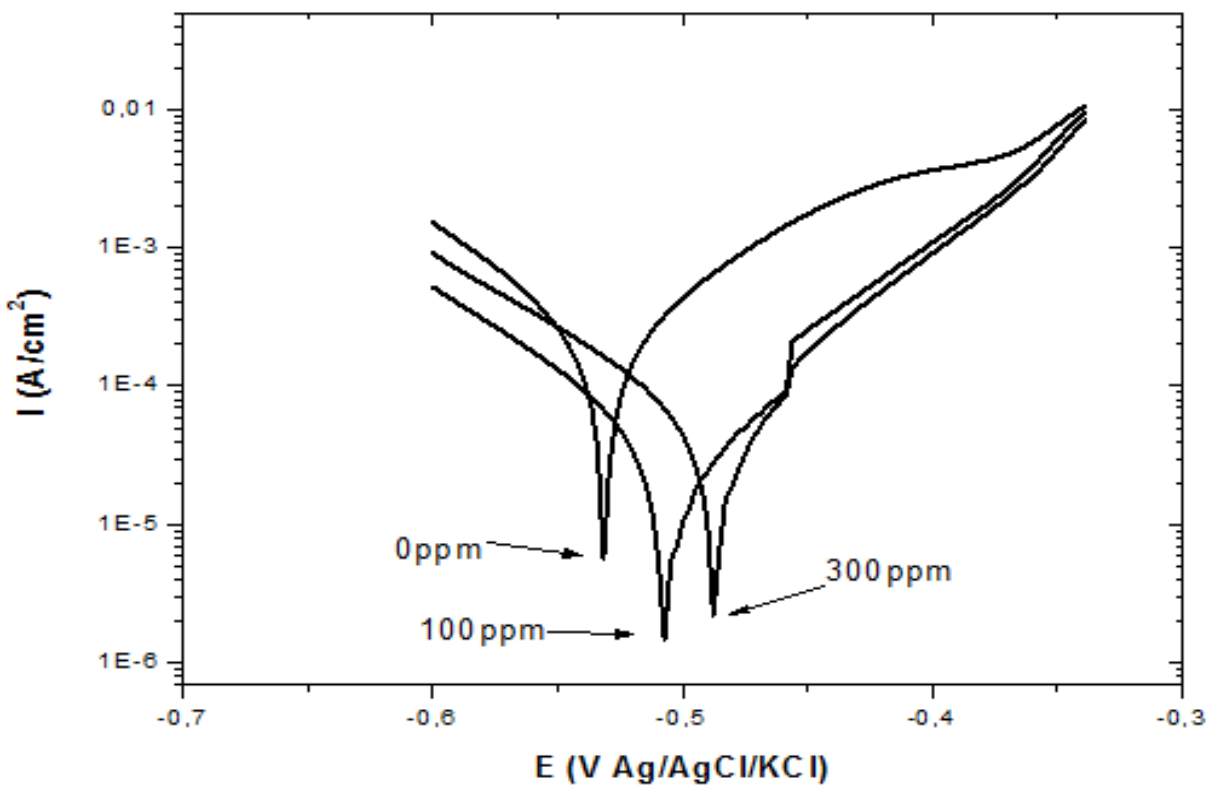

Figura 07: Curvas de polarização potenciodinâmica obtidas para aço - carbono em ácido clorídrico $0,5 \mathrm{~mol} \cdot \mathrm{L}^{-1}$ na ausência e presença de Mangiferina nas concentrações de 100 e 300 ppm à temperatura ambiente.

\section{DISCUSSÃO}

Observando a figura 02, para as amostras imersas apenas em meio ácido, o resultado de perda de massa apresentou uma média de 0,96 gramas em 144 horas de imersão, que seria cerca de 2,13\% da massa total da amostra ensaiada. Em comparação com as amostras que tiveram adição de concentrações conhecidas de Mangiferina (100 e 300 ppm), estas apresentaram uma redução da perda de massa significativa em relação à amostra na ausência de Mangiferina $(0 \mathrm{ppm})$. Na tabela 01 consta o resumo dos resultados de perda de massa relativa para cada concentração em estudo no tempo de 144 horas. 
Tabela 01: perda de massa em grama por concentração

\begin{tabular}{lc}
\hline Conc. & Massa relativa (\%) \\
\hline $0 p p m$ & 2,13 \\
\hline $100 p p m$ & 0,76 \\
\hline $300 p p m$ & 0,48 \\
\hline
\end{tabular}

Visualizando a tabela 01, observa-se uma redução significativa da perda de massa relativa com a adição de Mangiferina. A perda de massa para a maior concentração de Mangiferina (300 ppm) reduziu em $77,5 \%$ a ação corrosiva do meio, quando comparada com a amostra sem a adição de Mangiferina (0 ppm). Este resultado sugere que a Mangiferina apresenta comportamento inibidor no estudo proposto.

Os resultados apresentados acima estão coerentes com a literatura[18]. Segundo os autores BOUMHARA e et al, que elaboraram um estudo detalhado do efeito da inibição do óleo essencial Artemisia Mesatlantica (AMEO) sobre o comportamento de corrosão do aço-carbono em solução de ácido clorídrico (1 $\mathrm{mol} \cdot \mathrm{L}^{-1} \mathrm{HCl}$ ) a $30,8^{\circ} \mathrm{C}$. O estudo demostrou que o óleo essencial reduziu a perda de massa em $92 \%$ com uma concentração de $3 \mathrm{~g} / \mathrm{L}$ de AMEO.

Visualizando a figura 03, nota-se que os PCAs, após um intervalo de tempo de 10 min (600s) apresentam valores de potenciais praticamente constantes com os exibidos para um ensaio de até 1 hora (60 min.). Este resultado sugere que o sistema já se encontra estabilizado cineticamente para um tempo de imersão de 10 minutos[21].

Nas figuras 04 e 05 estão apresentados os diagramas de EIE no formato de Bode e Nyquist respectivamente para as concentrações de 0, 100 e 300 ppm de Mangiferina.

$\mathrm{Na}$ figura 04, de acordo com o diagrama de Bode, observa-se apenas um processo eletroquímico ocorrendo na superfície do eletrodo para um intervalo de frequência de $40.000 \mathrm{~Hz}$ até aproximadamente $1 \mathrm{~Hz}$, visto que o mesmo só apresenta uma constante de tempo[22].

$\mathrm{Na}$ figura 05 pode-se observar que a resistência do eletrólito $\left(\mathrm{R}_{\Omega}\right)$ na região de alta frequência é extremamente baixo sendo desprezível a polarização por queda ôhmica não sendo necessário ajustar os valores de densidade de corrente apresentados na figura 07[15]. Visualiza-se também um aumento dos valores de impedância com o aumento da concentração de Magiferina.

De acordo com os graficos da figura 06, observa-se um aumento dos valores de Rct com o aumento concentração de Mangiferina e a diminuição dos valores de C com o aumento da concentração de mangiferina. Tal resultado leva a crê que o inibidor está diminuindo o processo de transferência de carga sobre a superfície do substrato em estudo, reduzindo, cineticamente, o processo corrosivo para as amostras adicionadas do antioxidante. Tal redução da resistência a transferência de carga pode está associado a adsorção do inibidor Mangiferina na superficie eletródica. Este resultado está de acordo com a literatu$\operatorname{ra}[19][20]$.

Segundos os autores YADAV, SARKAR E PURKAIT (2015), que estudaram a ação inibidora de alguns compostos sintetizados de aminoácidos na corrosão do aço N80 numa solução de HCI a 15\% usando polarização potenciodinâmica e impedância AC mostraram que o inibidor em estudo apresentava uma boa eficiência de inibição. As medidas de impedância mostram que a resistência de transferência de carga (Rct) aumentou e a Capacitância da dupla camada elétrica $(\mathrm{Cdl})$ diminuiu com aumento da concentração dos inibidores. Este resultado foi associado, segundo os autores, a adsorção das moléculas do inibidor na superfície do aço N80, que foi comprovado pela técnica de Microscopia de Força Atômica (AFM) onde foi revelado processo adsortivo do inibidor sobre a superfície eletródica.

Os autores HAMDANI et al (2015), estudaram o efeito da inibição de alcaloides extraídos de Retama Monosperma (AERs) sobre o comportamento da corrosão do aço-carbono em solução de ácido clorídrico (1 $\left.\mathrm{mol} \cdot \mathrm{L}^{-1} \mathrm{HCl}\right)$ a temperatura de $30^{\circ} \mathrm{C}$. O estudo mostrou que os alcaloides extraídos das sementes de Retama Monosperma (AER) pode ser usado como um inibidor eficaz para a corrosão do aço-carbono em $1 \mathrm{~mol} \cdot \mathrm{L}^{-1} \mathrm{de}$ $\mathrm{HCl}$. A eficiência da inibição com o aumento da concentração do inibidor foi de aproximadamente $94 \%$, com $400 \mathrm{mg} / \mathrm{L}$. Os resultados de Espectroscopia de Impedância Eletroquímica indicaram que o valor da resistência de transferência de carga (Rct) aumentou e que a capacitância da dupla camada elétrica diminuiu. Este resultado foi atribuído ao aumento da espessura da dupla camada elétrica em consequência a adsorção do inibidor em estudo.

Visualizando as curvas de polarização expostas na figura 07, observa-se o deslocamento dos PCAs para valores mais catódicos com aumento da concentração de Mangiferina na solução ácida. Também, pode ser 
observado uma redução das densidades de corrente na região anódica com o aumento da concentração de mangiferina no meio ácido em relação à concentração de 0 ppm. Este resultado sugere que o inibidor proposto (Mangiferina) apresenta atividade inibitória no processo corrosivo do aço-carbono no sistema proposto, estando de acordo com os resultados obtidos de perda de massa apresentados e os resultados de espectroscopia de impedância eletroquímica. Pode ser observado também nas curvas polarização potenciodinâmica expostas na figura 05 que a ação do inibidor alterou os valores de densidades de corrente tanto na região catódica como na região anódica reduzindo-as. Este resultado, conforme apresentado na literatura, sugere que o inibidor em estudo tem ação mista, ou seja, sugere-se que a Mangiferina reduz as reações anódica e catódicas sobre a superfície eletródica[17].

\section{CONCLUSÃO}

Conclui-se que a Mangiferina apresenta propriedades inibidoras de corrosão dentro do sistema proposto quando comparadas a ausência total deste composto.

Os estudos realizados indicam que a Mangiferina apresenta mecanismo de inibição com ação mista.

\section{AGRADECIMENTOS}

Os autores agradecem aos Laboratórios de Materiais - LMAT e de Inspeção e Análise de Falhas - LIAF do IFCE e ao Laboratório de Química Orgânica da UFC pela realização dos experimentos, ao Grupo de Inspeção e Análise de Falhas - GIAF do IFCE pelo suporte técnico, além da CAPES, CNPq, FUNCAP pelo incentivo financeiro.

\section{BIBLIOGRAFIA}

[1] SOUZA, E.D. Análise de corrosão por meio de perda de massa e espessura em aços pela ação da água produzida de poços petrolíferos, In: Dissertação M.Sc, Departamento de Engenharia e Ciências de Materiais, Universidade Federal de Sergipe, 2010.

[2] GENTIL, V. Corrosão. Rio de Janeiro, Livros Técnicos e Científicos, $6^{\mathrm{a}}$ ed., 2013.

[3] SILVA, A.L.C.; MEI, P.R. Aços e ligas especiais. 2aed., São Paulo, SP, Edgard Blucher, pp. 646, 2006.

[4] DEFLORIAN, F., et al. "Study of the effect of corrosion inhibitors on powder coatings appliedon steel", Progress in Organic Coatings, v.77, pp. 2133 - 2139, 2014.

[5] AMIN, M. A. "Testing validity of the Tafel extrapolation method for monitoring corrosion of cold rolled steel in $\mathrm{HCl}$ solutions - Experimental and theoretical studies", Corrosion Science, v. 52, pp. 140 -151, 2010.

[6] EDDY, N.O., ODOEMELAM, S.A., "Inhibition of the corrosion of mild steel in HCl by sparfloxacin.", African Journal of Pure and Applied Chemistry, v. 2, pp. 132-138, 2008.

[7] EDDY, N.O., EBENSO, E.E., “Adsorption and inhibitive properties of ethanol extracts of Musa sapietum peels as a green corrosion inhibitor for mild steel in H2SO4", Afr. J. Pure Appl. Chem., v. 2, pp.46-54, 2008.

[8] AVWIRI, G.O., IGHO, F.O., "Inhibitive action of Vernonia amygdalina on the corrosion of aluminium alloys in acidic media", Mater. Lett, v. 57, pp.3705-3711, 2001.

[9] EL-ASHRY, E.H., EL-NEMIR, A., ESAWY, S.A., RAGAB, S., “Corrosion inhibitors part II: quantum chemical studies on the corrosion inhibitions of steel in acidic medium by some triazole, oxadiazole and thiadiazole derivatives”, Electrochimica Acta, v.51, pp.3957-3968, 2006.

[10] SETHURAN, M.G., RAJA, P.B., "Corrosion Inhibition of Mild Steel by Datura Metel in Acidic Medium.” Pigm. Resin Technol, v. 34, pp. 327-331, 2006.

[11] SAMPAIO, C. G. et al. "Chitosan/mangiferin particles for $\mathrm{Cr}(\mathrm{VI})$ reduction and removal.", International Journal of Biological Macromolecules, v. 78, pp. 273-279, 2015.

[12] QURAISHI, M.A., et al. "Green approach to corrosion inhibition of mild steel in hydrochloric acid and sulphuric acid solutions by the extract of Murraya koenigii leaves.", Materials Chemistry and Physics, v. 122, pp. 114-122, 2010.

[13] CANUTO, K. M., "Propriedades Químicas e Farmacológicas de Mangiferina: Um Composto Bioativo de Manga (Mangifera indica L.)", Embrapa, v. 21, pp. 218, 2009.

[14] RICHARDSON, P. M., "The taxonomic significance of C-glycosylxanthones in flowering plants", Biochemical Systematics and Ecology, Oxford, v. 11, pp. 371-375, 1983.

[15] TICIANELli, E. A., GONZALEZ, E. R. Eletroquímica: princípios e aplicações, São Paulo, Editora Edusp, 2 ed, pp.1, 2013. 
[16] BARros, I. B., MOSCOSO, H. Z. L., CUSTODIO, D. L., et al., "Casca Preciosa (Aniba canelilla) como Inibidor de Corrosão do Aço-Carbono.", Revista Virtual de Química - RVQ, http://www.uff.br/rvq, 2015.

[17] SHABANI-NOOSHABADI, M., GHANDCHI. M. S. "Santolina chamaecyparissus extract as a natural source inhibitor for 304 stainless steel corrosion in 3.5\% NaCl.", Journal of Industrial and Engineering Chemistry, v. 31, pp. 231-237, 2015.

[18] BOUMHARA, K., TABYAOUI, M., JAMA, C., BENTISS, F. "Artemisia Mesatlantica essential oil as green inhibitor for carbon steel corrosion in $1 \mathrm{M} \mathrm{HCl}$ solution: Electrochemical and XPS investigations", Journal of Industrial and Engineering Chemistry, v. 29, pp. 146-155, 2015.

[19] YADAV, M., SARKAR, T.K., PURKAIT, T. "Amino acid compounds as eco-friendly corrosion inhibitor for $\mathrm{N} 80$ steel in $\mathrm{HCl}$ solution: Electrochemical and theoretical approaches", Journal of Molecular Liquids, v. 212, pp. 731-739, 2015 .

[20] EL HAMDANI, N., FDIL, R., TOURABI, M., et al., “Alkaloids extract of Retama monosperma (L.) Boiss. seeds used as noveleco-friendly inhibitor for carbon steel corrosion in $1 \mathrm{M} \mathrm{HCl}$ solution: Electrochemical and surface studies", Applied Surface Science, v. 357, pp. 1294-1305, 2015.

[21] BARD, A.J., FAULKNER, L.R. Electrochemical methods: fundamentals and applications. USA, 2a ed, pp. 460, 2001.

[22] MACDONALD, J.R. Impedance spectroscopy - emphasizing solid materials and systems, New York, Wiley-interscience, $1^{\mathrm{a}}$ ed, 1987. 